PROCEEDINGS OF THE

AMERICAN MATHEMATICAL SOCIETY

Volume 134, Number 1, Pages 9-13

S 0002-9939(05)08272-9

Article electronically published on August 15, 2005

\title{
SHARPLY 2-TRANSITIVE GROUPS WITH POINT STABILIZER OF EXPONENT 3 OR 6
}

\author{
PETER MAYR
}

(Communicated by Jonathan I. Hall)

\begin{abstract}
Using the fact that all groups of exponent 3 are nilpotent, we show that every sharply 2 -transitive permutation group whose point stabilizer has exponent 3 or 6 is finite.
\end{abstract}

\section{INTRODUCTION}

Let $G$ be a sharply 2-transitive permutation group acting on a (possibly infinite) set $\Omega$, that is, $G$ is 2-transitive on $\Omega,|\Omega|>1$, and only the identity of $G$ fixes more than one element in $\Omega$.

It is still unknown whether an infinite sharply 2-transitive group $G$ always has a normal subgroup that is regular (i.e. sharply 1-transitive on $\Omega$ ) as in the finite case. Kerby gave an affirmative answer to that question under the assumption that for some $\alpha \in \Omega$ every conjugacy class in the point stabilizer $H:=\{g \in G \mid g(\alpha)=\alpha\}$ is finite [2, Theorem 9.6]. Suchkov showed that if $H$ is a 2-group, then $G$ has a regular normal subgroup and $G$ is in fact finite [5]. However it is not known whether a sharply 2-transitive group with point stabilizer of finite exponent is necessarily finite. In this note we prove the following:

Theorem 1.1. Let $G$ be a sharply 2-transitive permutation group on $\Omega$, and let $H$ be the stabilizer in $G$ of a point $\alpha \in \Omega$. Then we have:

(1) If $\exp H=3$, then $|G|=12$.

(2) If $\exp H=6$, then $|G|=42$.

Consequently every sharply 2-transitive group $G$ with point stabilizer of exponent 3 or 6 has a regular normal subgroup. In fact $G$ is the group of linear functions on the field of order 4 or 7 , respectively.

As a further consequence of Theorem 1.1, every near-field with multiplicative group of exponent 3 or 6 is a finite field. We note that every zero-symmetric nearring with 1 , whose elements satisfy $x^{n}=x$ for a fixed integer $n>1$, is a subdirect product of near-fields satisfying the same equation (cf. [3] or the corresponding result for rings by Jacobson [1). Hence, by Theorem 1.1, every zero-symmetric near-ring with 1 that satisfies $x^{7}=x$ is a subdirect product of finite fields. In

Received by the editors July 21, 2004.

2000 Mathematics Subject Classification. Primary 20B20; Secondary $20 B 22$.

Key words and phrases. (Infinite) sharply 2-transitive groups.

This work was supported by grant P15691 of the Austrian National Science Foundation (FWF) and was obtained during the author's visit at UW Madison, Wisconsin.

(C)2005 American Mathematical Society Reverts to public domain 28 years from publication 
particular both addition and multiplication are commutative operations for such a near-ring.

\section{Auxiliary RESUlts}

Following the exposition in [5] we state the basic results on sharply 2-transitive groups, which we will need to prove Theorem 1.1.

Throughout this section we use the following conventions. Let $G$ be a sharply 2 -transitive group on a set $\Omega$. We fix $\alpha, \beta \in \Omega, \alpha \neq \beta$, and we fix the stabilizer of $\alpha, H:=\{g \in G \mid g(\alpha)=\alpha\}$. Since $G$ is sharply 2 -transitive, we have a uniquely determined element $i \in G$ such that $i(\alpha)=\beta$ and $i(\beta)=\alpha$. We note that $i$ is an involution in $G \backslash H$.

Lemma 2.1 ([5, 1.1]). For all $g \in G \backslash H$, there are uniquely determined elements $a, b \in H$ such that $g=a i b$.

Lemma $2.2([5,1.2])$. All involutions in $G \backslash H$ are of the form $i^{a}$ for some $a \in H$, and all involutions in $G$ are conjugate in $G$.

Lemma 2.3 ([5, 1.3]). There is at most one involution in $H$.

We call an element $g \in G$ regular if $g(\gamma) \neq \gamma$ for all $\gamma \in \Omega$.

Lemma 2.4 ([5, 1.9]). Every product of 2 distinct involutions in $G$ is regular.

Lemma 2.5. Every non-trivial element in $G$ that commutes with a regular element is regular.

Proof. Let $g \in G$ be regular. Let $a \in G$ be such that $a$ commutes with $g$ and $a$ fixes some $\gamma \in \Omega$. Then $a=a^{g}$ stabilizes $\gamma$ and $g^{-1}(\gamma) \neq \gamma$. Thus $a=1$.

Lemma 2.6. Let $j \in H$ be an involution. If $H$ is torsion, then ij has finite order.

Proof. Since $j^{i} \notin H$, we have $a \in H$ such that $j^{i}=i^{a}$ by Lemma 2.2 By Lemma 2.3. $j$ is central in $H$. Hence $(i j)^{a}=i^{a} j=j^{i} j=(i j)^{2}$. Let $m$ denote the order of $a$. Then $i j=(i j)^{a^{m}}=(i j)^{2^{m}}$. The order of $i j$ divides $2^{m}-1$.

Let $\bar{H}:=H \backslash\left\{x \in H \mid x^{2}=1\right\}$. By Lemma 2.1, we may define functions $\varphi, \psi: \bar{H} \rightarrow H$ by

$$
\varphi(x):=y z \text { and } \psi(x):=z y,
$$

where $y, z \in H$ are such that $x^{i}=y i z$. From Lemmas 2.2, 2.3, and 2.4, we obtain that $\varphi(\bar{H}) \subseteq \bar{H}$ and $\psi(\bar{H}) \subseteq \bar{H}$.

Lemma $2.7([5,1.4])$. We have $\varphi(\varphi(x))=x$ for all $x \in \bar{H}$.

Proof. Let $x \in \bar{H}$, and let $y, z \in H$ be such that $x^{i}=y i z$. Now $y^{i}=x i z^{-1}$ and $z^{i}=y^{-1} i x$ yield $(y z)^{i}=x i z^{-1} y^{-1} i x$. Hence $x^{-1}(y z)^{i}$ is an involution, and it is not contained in $H$. By Lemma 2.2, we have $a \in H$ such that $x^{-1}(y z)^{i}=i^{a}$. Then $(y z)^{i}=x a^{-1} i a$ yields $\varphi(\varphi(x))=\varphi(y z)=x$.

Lemma 2.8 ([5, 1.8]). The map $\psi: \bar{H} \rightarrow \bar{H}$ is injective.

Proof. Let $x, r \in \bar{H}$, and let $y, z, s, t \in H$ be such that $x^{i}=y i z$ and $r^{i}=$ sit. We assume that $z y=t s$. Then $x^{i z^{-1}}=z y i=t s i=r^{i t^{-1}}$ and, consequently, $x^{i z^{-1} t i}=r$. Since $\alpha$ is the unique fixed point of $r$, we have $\left(i z^{-1} t i\right)^{-1}(\alpha)=\alpha$. Now $i z^{-1} t i$ stabilizes both $\alpha$ and $i(\alpha)$. Hence $z=t$. Thus we obtain $y=s$ and $x=r$. 
Lemma 2.9. Let $C$ be a finite conjugacy class in $H$ with $C \subseteq \bar{H}$. Then $C=$ $\psi(\varphi(C))$.

Proof. We note that $\varphi(x)$ and $\psi(x)$ are conjugate in $H$ for all $x \in \bar{H}$. Since $\varphi(\varphi(C))=C$ by Lemma 2.7 we then have $\psi(\varphi(C)) \subseteq C$. Equality follows from Lemma 2.8 since $C$ is finite and $\varphi$ is bijective.

\section{Proof of the theorem}

For proving Theorem 1.1, we will need the following fact, whose proof may be found in [4].

Lemma 3.1 ([4, cf. 12.3.5, 12.3.6]). All groups of exponent 3 are nilpotent.

Proof of Theorem 1.1(1). We use the notation of the previous section. Let $G$ be a sharply 2-transitive group with $\exp H=3$. We will see that the finiteness of $H$ follows from Lemma 3.1 once we have shown:

Every finite normal subgroup of $H$ has order at most 3 .

Let $\bar{H}:=H \backslash\{1\}$. First we show

$$
i \cdot i^{a}=i^{a^{2}} \text { for all } a \in \psi(\bar{H}) .
$$

Let $x \in \bar{H}$, and let $y, z \in H$ be such that $x^{i}=y i z$. The order of $x^{i z^{-1}}=z y i$ is 3 . For $a:=z y$, we have $1=(a i)^{3}=\left(a i a^{2}\right)\left(a^{-1} i a\right) i$. By $a=a^{-2}$, this yields (3.2).

For proving (3.1), we let $U$ be a finite normal subgroup of $H$ with $U \neq\{1\}$. We claim that

$$
K:=\left\{i^{u} \mid u \in U\right\} \cup\{1\} \text { is an elementary abelian 2-group. }
$$

It suffices to show that the product of any 2 distinct involutions in $K$ is contained in $K$. Since $U$ is finite and normal in $H$, all elements of $U$ have finite conjugacy classes in $H$. Hence $U \backslash\{1\} \subseteq \psi(\bar{H})$ by Lemma 2.9. For $u, v \in U, u \neq v$, we have $i^{u} \cdot i^{v}=\left(i \cdot i^{v u^{-1}}\right)^{u}=\left(i^{v u^{-1} v u^{-1}}\right)^{u}=i^{v u^{-1} v} \in K$, where we used (3.2) for $a=v u^{-1}$. Thus $K$ is a group. Since $\exp K=2$, we also have that $K$ is elementary abelian.

We note that $i^{u} \neq i^{v}$ for all $u, v \in U, u \neq v$, by Lemma 2.1. Thus

$$
|K|=|U|+1 \text { and }|K|=2^{n}
$$

for some number $n$. Since $U$ is a subgroup of $H$, we have that $|U|=2^{n}-1$ is a power of 3 . We note that 3 divides $2^{n}-1$ if and only if $n$ is even. Hence $2^{n}-1=\left(2^{n / 2}-1\right)\left(2^{n / 2}+1\right)$. Both $2^{n / 2}-1$ and $2^{n / 2}+1$ are powers of 3 . This implies $n=2$ and proves (3.1).

By Lemma 3.1, we have that $H$ is nilpotent and hence $|Z(H)|>1$. Together with (3.1) this yields $|Z(H)|=3$. Then all finite normal subgroups of $H / Z(H)$ are trivial by (3.1). Consequently the center of $H / Z(H)$ is trivial and $H=Z(H)$. Since $G$ is sharply 2-transitive on $\Omega$ and since $G$ has a point stabilizer of order 3, we have $|\Omega|=4$ and $|G|=4 \cdot 3$. Theorem 1.1(1) is proved.

Our strategy for proving Theorem 1.1(2) is similar to that for Theorem 1.1(1). The key step is to show that every finite abelian normal subgroup of a point stabilizer acts on some subgroup of regular elements in $G$. 
Proof of Theorem 1.1(2). We use the notation of the previous section. Let $G$ be a sharply 2-transitive group with $\exp H=6$. By Lemma 2.3. we have a unique involution $j$ in $H$. Hence the subgroup $\langle j\rangle$ that is generated by $j$ is central in $H$ and $\exp H /\langle j\rangle=3$. Since $\langle j\rangle$ is central and since $H /\langle j\rangle$ is nilpotent by Lemma 3.1. we have that $H$ is nilpotent. Let $P$ be a Sylow 3 -subgroup of $H$. By [4, 5.2.7], we have that

$$
H=P\langle j\rangle .
$$

In particular, $P$ is normal in $H$ and $P=\left\{x \in H \mid x^{3}=1\right\}$.

We write $\bar{H}:=H \backslash\{1, j\}$ and $\bar{P}:=P \backslash\{1\}$. We show that

$$
\psi(\bar{P}) \subseteq \bar{P} j .
$$

Let $x \in \bar{P}$, and let $y, z \in H$ be such that $x^{i}=y i z$. Seeking a contradiction, we suppose that $b:=z y$ has order 3 . We note that $\left(x^{i}\right)^{3}=1$ implies $(b i)^{3}=1$. By $b^{-1}=b^{2}$, we obtain $i \cdot i^{b}=i^{b^{2}}$. Since the involutions $i^{b^{2}}$ and $j$ are conjugate by Lemma 2.2 $i^{b^{2}}$ stabilizes exactly one point. But $i \cdot i^{b}$ is regular or 1 by Lemma 2.4 Thus we have a contradiction and $b^{3} \neq 1$. Since $b \in \bar{H}$ by the definition of $\psi$, we have $b \in \bar{P} j$, and (3.5) is verified.

Next we let $x \in \bar{H}$ and we prove:

$$
\text { If } \psi(x) \in \bar{P} j \text {, then } \psi(\psi(x)) \in \bar{P} \text {. }
$$

Let $y, z \in H$ be such that $x^{i}=y i z$. We assume that $z y \in \bar{P} j$. Let $v, w \in H$ be such that $\left(x^{2}\right)^{i}=v i w$. From $\left(x^{2}\right)^{i}=y i z y i z$ we obtain $i z y i=y^{-1} v i w z^{-1}$. We now consider $\psi(\psi(x))=\psi(z y)=w z^{-1} y^{-1} v$. Since $z^{-1} y^{-1}$ is conjugate to $(z y)^{-1} \in P j$, we have $z^{-1} y^{-1} \in P j$. Hence $\psi(\psi(x)) \in P j w v$. Since $x^{2} \in \bar{P}$, we have $w v \in P j$ by (3.5). Hence $\psi(\psi(x)) \in P j P j=P$. Since $\psi(\bar{H}) \subseteq \bar{H}$, (3.6) is proved.

Now let $C$ be a finite conjugacy class in $H$ with $C \subseteq \bar{P} j$. We show that

$$
C \subseteq \psi(\bar{P}) .
$$

By Lemma 2.9, we have $C=\psi(\varphi(C))$. Then $\psi(C)=\psi(\psi(\varphi(C))) \subseteq \bar{P}$ by (3.6). Since each element of $\varphi(C)$ is conjugate to an element of $\psi(C)$ by the definition of $\varphi$ and $\psi$, we have $\varphi(C) \subseteq \bar{P}$, and (3.7) is proved.

For $a \in \bar{P}$ such that the conjugacy class of $a$ is finite, we can now prove

$$
i j \cdot(i j)^{a} \cdot(i j)^{a^{2}}=1 .
$$

Since $a j \in \bar{P} j$ has a finite number of conjugates, we have $x \in \bar{P}$ and $y, z \in H$ such that $x^{i}=y i z$ and $z y=a j$ by (3.7). Now $x^{i z^{-1}}=a j i$ and $x^{3}=1$ imply $(a j i)^{3}=1$. Thus $1=i j\left(a^{-1} i j a\right)\left(a^{-2} i j a^{-1}\right)$, and (3.8) follows.

We are now ready to show:

Every finite abelian normal subgroup of $P$ has order at most 3.

To this end, we let $U$ be a finite abelian normal subgroup of $P$ with $U \neq\{1\}$. We claim that

$$
K:=\left\langle\left\{i^{u} j \mid u \in U\right\}\right\rangle \text { is abelian and finite. }
$$

To show that $K$ is abelian, it suffices to check that any 2 distinct generators commute. Let $u, v \in U, u \neq v$. Then we have $i^{u} j \cdot i^{v} j=\left(i j \cdot i^{v u^{-1}} j\right)^{u}=\left(j i^{v u^{-1} v u^{-1}}\right)^{u}=$ $j i^{v u^{-1} v}$, where we used (3.8) for $a=v u^{-1}$. Since $U$ is abelian of exponent 3, we obtain $i^{u} j \cdot i^{v} j=j i^{u^{2} v^{2}}$ and, by symmetry, $i^{v} j \cdot i^{u} j=j i^{v^{2} u^{2}}$. Thus $K$ is abelian. 
By Lemma 2.6. the order of $i^{u} j$ is finite for all $u \in U$. As an abelian group, which is generated by finitely many elements of finite order, $K$ is finite.

Since $i j \in K$ is regular by Lemma 2.4. all non-trivial elements in $K$ are regular by Lemma 2.5 Hence $K \cap H=\{1\}$ and, consequently, $U^{k} \cap U=\{1\}$ for all $k \in K \backslash\{1\}$. Thus $K U$ is a finite Frobenius group with Frobenius kernel $K$ and Frobenius complement $U$. Since $U$ is a 3 -group, $U$ is cyclic by [4, 10.5.6]. From $\exp U=3$ it follows that $|U|=3$. Hence (3.9) is proved.

From (3.9) together with Lemma 3.1, we obtain $|Z(P)|=3$ and that $P / Z(P)$ has a trivial center. We have $P=Z(P)$, and hence $|H|=6$ by (3.4). As a sharply 2 -transitive group with point stabilizer of order $6, G$ acts on a set $\Omega$ of size 7 and $|G|=7 \cdot 6$. Theorem 1.1(2) is proved.

\section{REFERENCES}

1. N. Jacobson. Structure theory for algebraic algebras of bounded degree. Ann. of Math. (2), 46:695-707, 1945. MR0014083 (7:238c)

2. W. Kerby. On infinite sharply multiply transitive groups. Vandenhoeck \& Ruprecht, Göttingen, 1974. Hamburger Mathematische Einzelschriften, Neue Folge, Heft 6. MR0384938 (52:5808)

3. S. Ligh. On the commutativity of near rings. II. Kyungpook Math. J., 11:159-163, 1971. MR0302708 (46:1852)

4. D. J. S. Robinson. A course in the theory of groups, volume 80 of Graduate Texts in Mathematics. Springer-Verlag, New York, second edition, 1996. MR.1357169 (96f:20001)

5. N. M. Suchkov. On the finiteness of some exactly doubly transitive groups. Algebra Logika, 40(3):344-351, 374, 2001. MR 1857888 (2002g:20004)

Institut für Algebra, Johannes Kepler Universität Linz, 4040 Linz, Austria

E-mail address: peter.mayr@algebra.uni-linz.ac.at 